\title{
ON PARABOLIC SUBMONOIDS OF A CLASS OF SINGULAR ARTIN MONOIDS
}

\author{
NOELLE ANTONY
}

(Received 11 April 2005; revised 17 August 2005)

Communicated by D. Easdown

\begin{abstract}
This paper concerns parabolic submonoids of a class of monoids known as singular Artin monoids. The latter class includes the singular braid monoid - a geometric extension of the braid group, which was created for the sole purpose of studying Vassiliev invariants in knot theory. However, those monoids may also be construed (and indeed, are defined) as a formal extension of Artin groups which, in turn, naturally generalise braid groups. It is the case, by van der Lek and Paris, that standard parabolic subgroups of Artin groups are canonically isomorphic to Artin groups. This naturally invites us to consider whether the same holds for parabolic submonoids of singular Artin monoids. We show that it is in fact true when the corresponding Coxeter matrix is of 'type $F C$ '; hence generalising Corran's result in the 'finite type' case.
\end{abstract}

2000 Mathematics subject classification: primary 20M05, $20 \mathrm{~F} 36$.

Keywords and phrases: singular Artin monoids, parabolic submonoids.

\section{Preliminaries}

We begin with some formal definitions. Let $I$ be a finite indexing set, and let $M=\left(m_{i j}\right)_{i, j \in l}$ denote the matrix, indexed by the elements of $I$, that satisfies:

(i) $m_{i i}=1$ if $i \in I$;

(ii) $m_{i j}=m_{j i} \in\{2,3,4, \ldots, \infty\}$ whenever $i, j \in I$ and $i \neq j$.

Such a matrix is known as a Coxeter matrix. Every Coxeter matrix $M$ may be associated with a graph $\Gamma^{M}$ defined as follows:

The author thanks Dr David Easdown for his encouragement and many helpful discussions. The majority of the work in this article was undertaken while the author was at the University of Sydney's School of Mathematics and Statistics.

(C) 2007 Australian Mathematical Society 1446-7887/07 \$A2.00+0.00 
(i) $I$ is the set of vertices of $\Gamma^{M}$;

(ii) any two nodes $i, j \in I$ are joined by an edge if $m_{i j} \geq 3$;

(iii) the edge joining two vertices $i$ and $j$ is labelled by $m_{i j}$ if $m_{i j} \geq 4$; edge labels are suppressed whenever $m_{i j}=3$.

Such a graph is referred to as a Coxeter graph of type $M$. Now let $S=\left\{\sigma_{i} \mid i \in I\right\}$ be a set in one-to-one correspondence with $I$. If $X$ is a set, then $X^{*}$ denotes the free monoid generated by $X$. If $q$ is a natural number and $i, j \in I$ then $\left\langle\sigma_{i} \sigma_{j}\right\rangle^{q}$ indicates the alternating product $\sigma_{i} \sigma_{j} \sigma_{i} \cdots$ of length $q$ (that is, with $q$ factors). The Artin group of type $M, G_{M}$, is the group generated by $S$ subject to the relations

$$
\left\langle\sigma_{i} \sigma_{j}\right\rangle^{m_{i j}}=\left\langle\sigma_{j} \sigma_{i}\right\rangle^{m_{i j}} \quad \text { for } i, j \in I, m_{i j} \neq \infty \text {; }
$$

these relations are denoted by $\mathscr{R}_{1}$ and called the braid relations. In arguments below we regard a relation formally as an ordered pair of words. For example, the relation $\sigma_{i} \sigma_{j}=\sigma_{j} \sigma_{i}$ becomes the ordered pair $\left(\sigma_{i} \sigma_{j}, \sigma_{j} \sigma_{i}\right)$. If $X$ is a set of ordered pairs of words then $X^{\Sigma}=\{(U, V) \mid(U, V)$ or $(V, U) \in X\}$. The Coxeter group of type $M$, $W_{M}$, is the group generated by $S$ subject to the preceding braid relations $\mathscr{R}_{1}$, together with the relations $\sigma_{i}^{2}=1$ for every $i$ in $I$. Hence, Coxeter groups arise as quotient groups of Artin groups. If $W_{M}$ is finite then $M$ (or $\Gamma^{M}$ ) is said to be of finite type or spherical type. A Coxeter group is finite precisely when its graph is a finite disjoint union of the graphs shown in Figure 1 (see, for example, [14, 21]) .

The first, and arguably the most well-known, (non-abelian) example of an Artin group is the braid group established in 1925 by Artin [3]; thus the terminology Artin group suggested by Brieskorn and Saito [9]. Indeed, Artin groups are also known as generalised braid groups. Observe that $\mathcal{B}_{n+1}$, the braid group on $n+1$ strings, arises from the special case when $I=\{1, \ldots, n\}, m_{i j}=3$ when $|i-j|=1$, and $m_{i j}=2$ when $|i-j| \geq 2$. Its associated Coxeter graph is referred to as type $A_{n}$ (shown in Figure 1), and the corresponding Coxeter group is the symmetric group on $n+1$ letters.

We now extend Artin groups as follows [11, 19]: put $T=\left\{\tau_{i} \mid i \in I\right\}$, and let $S^{-1}=\left\{\sigma_{i}^{-1} \mid i \in I\right\}$, the set of formal inverses of $S$. The singular Artin monoid of type $M$, denoted by $\mathcal{S} G_{M}$, is the monoid generated by $S \cup S^{-1} \cup T$ and has as its defining relations the set $\mathscr{R}$, which is comprised of the free group relations $\sigma_{i} \sigma_{i}^{-1}=\sigma_{i}^{-1} \sigma_{i}=1$, the braid relations $\mathscr{R}_{1}$, and the relations $\mathscr{R}_{2}$ listed below:

$$
\begin{aligned}
\tau_{i} \sigma_{i} & =\sigma_{i} \tau_{i} \quad \text { for all } i \text { in } I ; \\
\tau_{i}\left\langle\sigma_{j} \sigma_{i}\right\rangle^{m_{i j}-1} & = \begin{cases}\left\langle\sigma_{j} \sigma_{i}\right\rangle^{m_{i j}-1} \tau_{j} & \text { if } m_{i j}<\infty \text { and is odd, or } \\
\left\langle\sigma_{j} \sigma_{i}\right\rangle^{m_{i j}-1} \tau_{i} & \text { if } m_{i j}<\infty \text { and is even; }\end{cases} \\
\tau_{i} \tau_{j} & =\tau_{j} \tau_{i} \quad \text { if } m_{i j}=2 .
\end{aligned}
$$




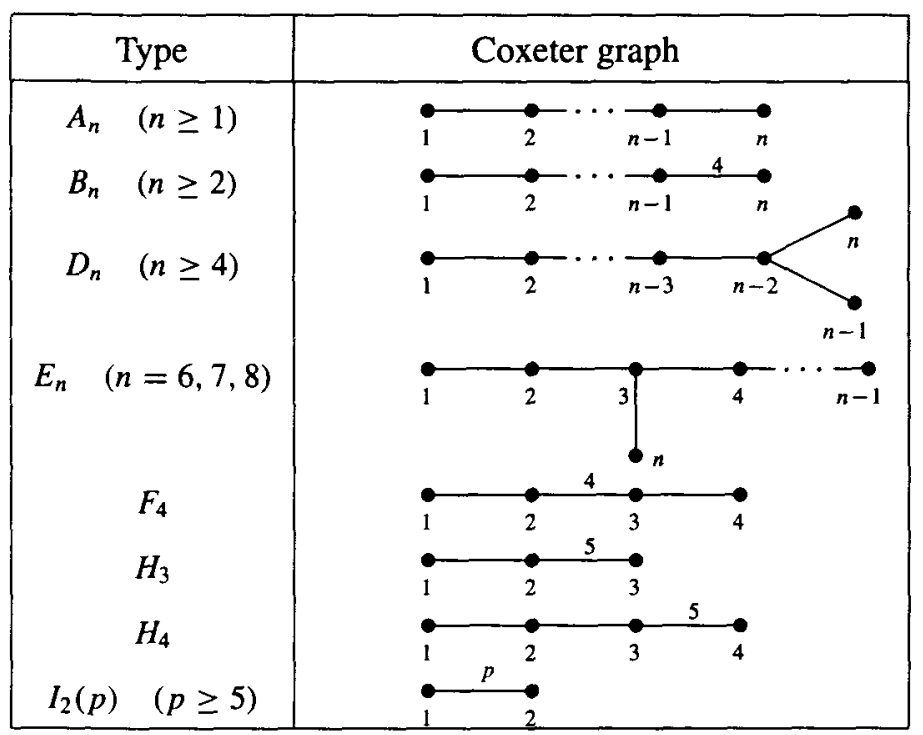

FIGURE 1. The irreducible Coxeter graphs of finite type. Unlabelled edges have value 3.

We define the positive singular Artin monoid of type $M$ to be the monoid generated by $S \cup T$ and the set of defining relations comprised of both $\mathscr{R}_{1}$ and $\mathscr{R}_{2}$ listed above.

REMARK 1. The special case when the singular Artin monoid is of type $A_{n}$ may be familiar to some readers as the singular braid monoid on $n+1$ strings, $\mathcal{S B}_{n+1}$, which was introduced by Baez [4] and Birman [8] in their study of knot invariants. We remark that, although singular Artin monoids are defined (abstractly) by the above generators and relations, $\mathcal{S B}_{n+1}$ was originally introduced geometrically in $[4,8]$ and was then shown (in [8, Lemma 3] and a subcase of [20, Theorem 2.1]) to admit the preceding presentation.

Where it does not cause confusion, elements of $G_{M}$ and $\mathcal{S} G_{M}$ may be referred to by words which represent them. If $A$ and $B$ are elements of $\left(S \cup T \cup S^{-1}\right)^{*}$, we write $A \approx B$ if $A$ can be transformed into $B$ by the use of the set of defining relations of $\mathcal{S} G_{M}$, and $A=B$ if the two words are equal letter by letter.

\section{Parabolic subgroups and submonoids}

Now let $J$ be any subset of $I$. Recall that $M$ is a Coxeter matrix over the finite indexing set $I$. Denote by $M_{J}$ the submatrix of $M$ containing the entries indexed by $J$; it is clear that $M_{J}$ is also a Coxeter matrix. In accordance with Corran [12, Section 5], we use the notation: $S_{J}=\left\{\sigma_{j} \mid j \in J\right\}, S_{J}^{-1}=\left\{\sigma_{j}^{-1} \mid j \in J\right\}, T_{J}=\left\{\tau_{j} \mid j \in J\right\}$. 
We denote by $\mathscr{R}_{1}$, and $\mathscr{R}_{J}$ the defining relations of $G_{M}$, and $\mathcal{S} G_{M_{J}}$ respectively. Then by the definition of these relations it is evident that $\mathscr{R}_{1} \subseteq \mathscr{R}_{1}$ and $\mathscr{R}_{J} \subseteq \mathscr{R}$. The subgroups of $W_{M}$ and $G_{M}$ generated by $S_{J}$ are denoted by $W_{M}^{J}$ and $G_{M}^{J}$ and are called the standard parabolic subgroups of $W_{M}$ and $G_{M}$ respectively. Let $P_{J}$ denote the submonoid of $\mathcal{S} G_{M}$ generated by $S_{J} \cup S_{J}^{-1} \cup T_{J}$; that is, the set of equivalence classes of words over $S_{J} \cup S_{J}^{-1} \cup T_{J}$ under $\approx$. Then $P_{J}$ is referred to as the parabolic submonoid defined by $J\left[12\right.$, Section 5]. Notice that $G_{M}^{J}$ is a homomorphic image of the Artin group $G_{M_{j}}$. Lek [23] and Paris [24] showed that this homomorphism is an isomorphism. This result was first discovered for Artin groups of finite type in $[9,15]$ (it was also later proved in [10]); for 'extra-large' type Artin systems in [2]; and was gradually extended to include all types in [23, 24]. It is also well-known that the subgroup $W_{M}^{J}$ is canonically isomorphic to the Coxeter group associated with the matrix $M_{J}$; its graph $\Gamma^{M_{J}}$ is the full subgraph of $\Gamma^{M}$ generated by $S_{J}$.

An analogous result holds for singular Artin monoids of finite type: namely, that $\mathcal{S} G_{M}$, naturally injects into $\mathcal{S} G_{M}$ whenever $M$ is of finite type, so that the image of that embedding is precisely $P_{J}[12$, Proposition 33$]$. Hence the following holds.

THEOREM 2 (Corran [12, Theorem 34]). Parabolic submonoids of singular Artin monoids of finite type are (isomorphic to) singular Artin monoids.

The Coxeter matrix $M$ is said to be right-angled if

$$
m_{i j} \in\{2, \infty\} \text { for } i, j \in I, i \neq j .
$$

Right-angled Artin groups are also known as graph groups or free partially commutative groups [19]. Their applications extend to areas such as random walks, parallel computation and cohomology of groups (see, for example, [7]). The Coxeter matrix $M$ is said to be of type $F C$ if it satisfies the ensuing condition:

- For every $J \subseteq I$, either $W_{M}$ is finite or $m_{s t}=\infty$ for some $s, t \in J, s \neq t$.

For example, the Coxeter group associated with the graph shown in Figure 2 is of type $F C$ [18]. The terminology $F C$ refers to 'flag complex'; it is introduced in [10] where the reader can find a detailed exposition and classification of such types.

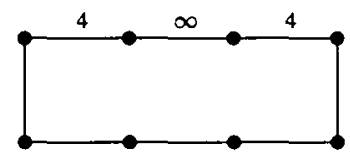

FIGURE 2.

REMARK 3. Observe that both right-angled and finite type Artin groups are of type $F C$. Furthermore, if $M$ is of type $F C$ and $J \subseteq I$, then $M_{J}$ (the submatrix of $M$ containing the entries indexed by $J$ ) is also a Coxeter matrix of type $F C$. 
In [12, Section 5], Corran postulates that although it is not clear how to generalise Theorem 2 to include singular Artin monoid of all types, she suspects that it does hold for arbitrary types. The object of this paper is to extend this theorem of Corran to singular Artin monoids of type $F C$. That is, we prove the following result.

THEOREM 4. Parabolic submonoids of singular Artin monoids of type FC are (isomorphic to) singular Artin monoids.

Except when explicitly stated, we assume throughout this paper that $M$ is of any type. If $V$ and $W$ are words over $S_{J} \cup S_{J}^{-1} \cup T_{J}$ and represent the same element of $\mathcal{S} G_{M_{J}}$, write $V \approx_{J} W$. By [23, 24], we have the following.

THEOREM 5. Let $U, V$ be words over $S_{J} \cup S_{J}^{-1}$ such that $U \approx V$. Then $U \approx{ }_{J} V$.

In [16], it was shown that the singular braid monoid on $n+1$ strings (that is, the singular Artin monoid of type $A_{n}$ ) can be embedded in a group. The group constructed by the authors relies heavily on the geometry of singular braids in space; more specifically, it has a geometric interpretation as singular braids with two types of (cancelling) singularities. By employing purely algebraic methods, Paris [25] gave another proof of the fact that singular braid monoids inject into groups. In fact, all singular Artin monoids embed into groups. This was shown (chronologically and with completely different proofs) in $[5,22,19]$. An evident corollary of this is that left and right cancellation hold in $\mathcal{S} G_{M}$.

Proposition 6. Let $C, W, V$ be words over $S \cup S^{-1} \cup T$ such that either $C W \approx C V$ or $W C \approx V C$. Then $W \approx V$.

The next proposition is a subcase of what is known as the 'FRZ' property [19, Proposition 4.1]. The property was first discovered in [17, Theorem 7.1] for the singular braid monoid on $n+1$ strings (defined in Remark 1); it was later shown to hold for singular Artin monoids of finite type [12, Theorem 31] and of type $F C$ [19, Proposition 4.1]. By [1, Appendix], the FRZ property holds for positive singular Artin monoids of any type.

PROPOSITION 7. Assume $M$ is of type FC. Let $U$ be a word over $S \cup S^{-1} \cup T$, $s, t \in I$ and suppose $\sigma_{s} U \approx U \sigma_{t}$. Then $\tau_{s} U \approx U \tau_{t}$.

\section{Proof of the main theorem}

Let $U, V$ be words over $S \cup S^{-1} \cup T$. We say $U$ and $V$ differ by an elementary transformation if there are words $X$ and $Y$ and a relation $\left(\kappa_{1}, \kappa_{2}\right) \in\left(\mathscr{R}_{1} \cup \mathscr{R}_{2}\right)^{\Sigma}$ such 
that $V=X \kappa_{1} Y$ and $U=X \kappa_{2} Y$. We say that a word $V$ is obtained from $U$ by a trivial insertion if there are words $X, Y$ and a letter $a \in S \cup S^{-1}$ such that $U=X Y$ and $V=X a a^{-1} Y$. In this case we also say that $U$ is obtained from $V$ by a trivial deletion.

Define a monoid homomorphism $\mathcal{N}$ from $\mathcal{S} G_{M}$ to $(\mathbb{Z},+)$ by

$$
\mathcal{N}: \sigma_{i}^{ \pm 1} \mapsto 0, \tau_{i} \mapsto 1 \text { for } i \in I .
$$

Thus $\mathcal{N}$ counts the number of taus in any given word. Now let $W$ be a word over $S \cup S^{-1} \cup T$, and suppose $\mathcal{N}(W)=k \geq 1$. Then there are words $W_{i}$ over $S \cup S^{-1}$ and generators $\tau_{a_{i}} \in T$ such that $W=W_{0} \tau_{a_{1}} W_{1} \tau_{a_{2}} \cdots W_{k-1} \tau_{a_{k}} W_{k}$. For $r=1, \ldots, k$, let

$$
\rho_{r}(W)=W_{0} \tau_{a_{1}} W_{1} \cdots \tau_{a_{r-1}} W_{r-1} \sigma_{a_{r}} W_{r} \tau_{a_{r+1}} W_{r+1} \cdots \tau_{a_{k}} W_{k}
$$

and

$$
\theta_{r}(W)=W_{0} \tau_{a_{1}} W_{1} \cdots \tau_{a_{r-1}} W_{r-1} W_{r} \tau_{a_{r+1}} W_{r+1} \cdots \tau_{a_{k}} W_{k} .
$$

Hence both $\rho_{r}$ and $\theta_{r}$ reduce the number of taus of $W$ by 1 . We observe that $\rho_{r}$ has been previously defined in, for example, $[6,13]$.

LEMMA 8. Let $W, V$ be words over $S \cup S^{-1} \cup T$ such that $W \approx V$, and suppose $\mathcal{N}(W)=k$ is at least 1 . Then for every $r \in\{1, \ldots, k\}$ there exists an $s \in\{1, \ldots, k\}$ such that $\rho_{r}(W) \approx \rho_{s}(V)$ and $\theta_{r}(W) \approx \theta_{s}(V)$.

PROOF. Let $r$ be any integer such that $1 \leq r \leq k$. Since $W \approx V$, there is a sequence $Z_{1}, \ldots, Z_{t}$ of words over $S \cup S^{-1} \cup T$ such that $W=Z_{1} \approx Z_{2} \approx \ldots \approx Z_{t}=V$ and $Z_{i+1}$ is obtained from $Z_{i}$ by an elementary transformation or by a trivial deletion or insertion. If $t=1$ the result is trivial and hence starts an induction. Suppose then that $t$ is least 2 . If $Z_{2}$ is obtained from $Z_{1}$ by a trivial deletion or insertion, it is evident that $\rho_{r}\left(Z_{1}\right) \approx \rho_{r}\left(Z_{2}\right)$ and $\theta_{r}\left(Z_{1}\right) \approx \theta_{r}\left(Z_{2}\right)$. So assume that $Z_{1}$ and $Z_{2}$ differ by an elementary transformation. If the relation involves any $\sigma$, then inspection of $\mathscr{R}_{1} \cup \mathscr{R}_{2}$ gives $\rho_{r}\left(Z_{1}\right) \approx \rho_{r}\left(Z_{2}\right)$ and $\theta_{r}\left(Z_{1}\right) \approx \theta_{r}\left(Z_{2}\right)$. Hence suppose the relation is of the form $\left(\tau_{i} \tau_{j}, \tau_{j} \tau_{i}\right)$ where $m_{i j}=2$. Then we see that either

$$
\rho_{r}\left(Z_{1}\right) \approx \rho_{r+1}\left(Z_{2}\right) \quad \text { and } \quad \theta_{r}\left(Z_{1}\right)=\theta_{r+1}\left(Z_{2}\right)
$$

or

$$
\rho_{r}\left(Z_{1}\right) \approx \rho_{r}\left(Z_{2}\right) \quad \text { and } \quad \theta_{r}\left(Z_{1}\right) \approx \theta_{r}\left(Z_{2}\right)
$$

or

$$
\rho_{r}\left(Z_{1}\right) \approx \rho_{r-1}\left(Z_{2}\right) \quad \text { and } \quad \theta_{r}\left(Z_{1}\right)=\theta_{r-1}\left(Z_{2}\right) .
$$

Thus there exists an integer $q \in\{1, \ldots, k\}$ such that

$$
\rho_{r}\left(Z_{1}\right) \approx \rho_{q}\left(Z_{2}\right) \quad \text { and } \quad \theta_{r}\left(Z_{1}\right) \approx \theta_{q}\left(Z_{2}\right) .
$$


By the inductive hypothesis, we deduce that $\rho_{q}\left(Z_{2}\right) \approx \rho_{s}\left(Z_{t}\right)$ and $\theta_{q}\left(Z_{2}\right) \approx \theta_{s}\left(Z_{t}\right)$ for some $s \in\{1, \ldots, k\}$, whence $\rho_{r}(W)=\rho_{r}\left(Z_{1}\right) \approx \rho_{q}\left(Z_{2}\right) \approx \rho_{s}\left(Z_{t}\right)=\rho_{s}(V)$, and similarly $\theta_{r}(W) \approx \theta_{s}(V)$, as required. The result now follows by induction.

THEOREM 9. Suppose $M$ is of type FC. Let $J \subseteq I$, and suppose $U \approx V$ where $U$ and $V$ are words over $S_{J} \cup S_{J}^{-1} \cup T_{J}$. Then $U \approx_{J} V$.

PROOF. Let $U, V$ be words over $S_{J} \cup S_{J}^{-1} \cup T_{J}$ such that $U \approx V$, and put $\mathcal{N}(U)=k$. If $k=0$, the result follows by Theorem 5 and starts an induction. So suppose $k \geq 1$. Then there exists an $a \in J$ and words $X_{1}, X_{2}$ over $S_{J} \cup S_{J}^{-1}$ and $S_{J} \cup S_{J}^{-1} \cup T_{J}$ respectively such that $U=X_{1} \tau_{a} X_{2}$. Thus

$$
X_{1} \tau_{a} X_{2}=U \approx V,
$$

so by Lemma 8 , there exists an $r \in\{1, \ldots, k\}$ such that

$$
X_{1} \sigma_{a} X_{2}=\rho_{1}(U) \approx \rho_{r}(V) \text { and } X_{1} X_{2}=\theta_{1}(U) \approx \theta_{r}(V) .
$$

Since $\mathcal{N}(U)=\mathcal{N}(V)=k \geq 1$, there are words $Y_{1}, Y_{2}$ over $S_{J} \cup S_{J}^{-1} \cup T_{J}$ and a generator $\tau_{b} \in T_{J}$ such that

$$
V=Y_{1} \tau_{b} Y_{2}, \quad \text { where } \mathcal{N}\left(Y_{1}\right)=r-1 \text { and } \mathcal{N}\left(Y_{2}\right)=k-r .
$$

Then

$$
\rho_{r}(V)=Y_{1} \sigma_{b} Y_{2} \quad \text { and } \quad \theta_{r}(V)=Y_{1} Y_{2}
$$

so by (2),

$$
X_{1} \sigma_{a} X_{2} \approx Y_{1} \sigma_{b} Y_{2} \text { and } X_{1} X_{2} \approx Y_{1} Y_{2}
$$

By noting that $X_{1}$ is over $S_{J} \cup S_{J}^{-1}$, we deduce that $X_{1}^{-1}$ is also over $S_{J} \cup S_{J}^{-1}$, so by (4), we obtain

$$
\sigma_{a} X_{2} \approx X_{1}^{-1} Y_{1} \sigma_{b} Y_{2} \quad \text { and } \quad X_{2} \approx X_{1}^{-1} Y_{1} Y_{2}
$$

moreover by (3), we see that

$$
k-1=\mathcal{N}\left(Y_{1} Y_{2}\right)=\mathcal{N}\left(X_{1}^{-1} Y_{1} Y_{2}\right) \geq \mathcal{N}\left(Y_{1}\right)=\mathcal{N}\left(\sigma_{a} X_{1}^{-1} Y_{1}\right) .
$$

Observe that by (5), $\sigma_{a} X_{1}^{-1} Y_{1} Y_{2} \approx \sigma_{a} X_{2} \approx X_{1}^{-1} Y_{1} \sigma_{b} Y_{2}$, so by Proposition 6,

$$
\sigma_{a} X_{1}^{-1} Y_{1} \approx X_{1}^{-1} Y_{1} \sigma_{b}
$$


Since $a, b \in J$, and $X_{1}^{-1}, X_{2}, Y_{1}, Y_{2}$ are all words over $S_{J} \cup S_{J}^{-1} \cup T_{J}$, (6) and (7) together with the inductive hypothesis give

$$
\sigma_{a} X_{1}^{-1} Y_{1} \approx_{J} X_{1}^{-1} Y_{1} \sigma_{b}
$$

furthermore, by (6) and the inductive hypothesis applied to the second equivalence of (5), we also infer that

$$
X_{2} \approx_{J} X_{1}^{-1} Y_{1} Y_{2}
$$

By Remark 3, $M_{J}$ is also of type $F C$; thus Proposition 7 may be applied to (8), and this yields

$$
\tau_{a} X_{1}^{-1} Y_{1} \approx_{J} X_{1}^{-1} Y_{1} \tau_{b}
$$

Hence

$$
\begin{aligned}
& U=X_{1} \tau_{a} X_{2} \quad \text { by (1) } \\
& \approx_{J} X_{1} \tau_{a} X_{1}^{-1} Y_{1} Y_{2} \quad \text { by (9) } \\
& \approx_{J} X_{1} X_{1}^{-1} Y_{1} \tau_{b} Y_{2} \quad \text { by (10) } \\
& \approx_{J} Y_{1} \tau_{b} Y_{2}=V \quad \text { by (3). }
\end{aligned}
$$

The result now follows by induction.

Proof of Theorem 4. Suppose $M$ is of type $F C$. Recall that $P_{J}$ denotes the submonoid of $\mathcal{S} G_{M}$ generated by $S_{J} \cup S_{J}^{-1} \cup T_{J}$. By Theorem $9, \mathcal{S} G_{M_{J}}$ naturally embeds in $S G_{M}$ with image $P_{J}$. Hence the parabolic submonoid $P_{J}$ is canonically isomorphic to the singular Artin monoid $S G_{M_{S}}$.

REMARK 10. The reader may notice that the only part of the proof of Theorem 9 that requires the $F C$ condition is Proposition 7. Hence in order to strengthen Theorem 4 to show that it holds for singular Artin monoids of arbitrary type it suffices to prove Proposition 7 for any Coxeter matrix $M$; the proof would then proceed unmodified to that of Theorem 9.

\section{References}

[1] N. Antony, 'On singular Artin monoids and contributions to Birman's conjecture', Comm. Algebra 33 (2005), 4043-4056.

[2] K. I. Appel and P. E. Schupp, 'Artin groups and infinite Coxeter groups', Invent. Math. 72 (1983), 201-220. 
[3] E. Artin, 'Theorie der Zöpfe', Abh. Math. Sem. Univ. Hamburg 4 (1926), 47-72.

[4] J. Baez, 'Link invariants of finite type and perturbation theory', Lett. Math. Phys. 26 (1992), 43-51.

[5] G. Basset, 'Quasi-commuting extensions of groups', Comm. Algebra 28 (2000), 5443-5454.

[6] P. Bellingeri, 'Centralisers in surface braid groups', Comm. Algebra 32 (2004), 4099-4115.

[7] M. Bestvina and N. Brady, 'Morse theory and finiteness properties of groups', Invent. Math. 129 (1997), 445-470.

[8] J. S. Birman, 'New points of view in knot theory', Bull. Amer. Math. Soc. (N.S.) 28 (1993), 253-286.

[9] E. Brieskorn and K. Saito, 'Artin-Gruppen und Coxeter-Gruppen', Invent. Math. 17 (1972), 245 271.

[10] R. Charney and M. W. Davis, 'The $K(\pi, 1)$-problem for hyperplane complements associated to infinite reflection groups', J. Amer. Math. Soc. 8 (1995), 597-627.

[11] R. Corran, 'A normal form for a class of monoids including the singular braid monoid', J. Algebra 223 (2000), 256-282.

[12] _ _ 'Conjugacy in singular Artin monoids', J. Aust. Math. Soc. 79 (2005), 183-212.

[13] J. Daz-Cantos, J. Gonzalez-Meneses and J. M. Tornero, 'On the singular braid monoid of an orientable surface', Proc. Amer. Math. Soc. 132 (2004), 2867-2873.

[14] P. de la Harpe, 'An invitation to Coxeter groups', in: Group Theory from a Geometrical Viewpoint, (ICTP, Trieste, Italy, 1990) (World Scientific, River Edge, NJ, 1991) pp. 193-253.

[15] P. Deligne, 'Les immeubles des groupes de tresses généralisés', Invent. Math. 17 (1972), 273-302.

[16] R. Fenn, E. Keyman and C. Rourke, 'The singular braid monoid embeds in a group', $J$. Knot Theory Ramifications 7 (1998), 881-892.

[17] R. Fenn, D. Rolfsen and J. Zhu, 'Centralizers in the braid group and the singular braid monoid', Enseign. Math. (2) 42 (1996), 75-96.

[18] E. Godelle, 'Parabolic subgroups of artin groups of type $F C$ ', Pacific J. Math. 208 (2003), 243-254.

[19] E. Godelie and L. Paris, 'On singular Artin monoids', in: Geometric methods in group theory, Contemporary Math. 372 (Amer. Math. Soc., Providence, RI, 2005) pp. 43-57.

[20] J. González-Meneses, 'Presentations for the monoids of singular braids on closed surfaces', Comm. Algebra 30 (2002), 2829-2836.

[21] J. E. Humphreys, Reflection groups and Coxeter groups, Cambridge Studies in Advanced Mathematics 29 (Cambridge Univ. Press, Cambridge, UK, 1990).

[22] E. Keyman, 'A class of monoids embeddable in a group', Turkish J. Math. 25 (2001), 299-305.

[23] $\mathrm{H}$. van der Lek, The homotopy type of complex hyperplane complements (Ph.D. Thesis, University of Nijmegen, The Netherlands, 1983).

[24] L. Paris, 'Parabolic subgroups of Artin groups', J. Algebra 196 (1997), 369-399.

[25] — - 'The proof of Birman's conjecture on singular braid monoids', Geom. Topol. 8 (2004), $1281-1300$.

School of Mathematics and Statistics

F07

The University of Sydney

NSW 2006

Australia

e-mail: noellea@maths.usyd.edu.au 